\title{
Recent Results from the Pierre Auger Observatory
}

\author{
Karl-Heinz Kampert* for the Pierre Auger Collaboration ${ }^{\dagger}$ \\ Bergische Universität Wuppertal, Department of Physics, 42119 Wuppertal (Germany) \\ E-mail: kampert@uni-wuppertal.de
}

Recent results from the Pierre Auger Observatory about the energy spectrum, mass composition, and arrival directions of ultra-high energy cosmic rays are reported. The ankle at $4 \cdot 10^{18} \mathrm{eV}$ and a suppression above $3 \cdot 10^{19} \mathrm{eV}$ consistent with the GZK effect is observed. At energies above $5.5 \cdot 10^{19} \mathrm{eV}$ a directional correlation of cosmic rays with the positions of nearby extragalactic objects is found, including an excess of events around the direction of Centaurus A, the nearest radio loud active galaxy. Measurements of the depth of shower maximum and its shower-byshower fluctuations suggest a proton dominated composition up to the ankle and a gradual change towards heavier masses with increasing energy. Shower fluctuations at the highest energies, 4 . $10^{19} \mathrm{eV}$, approach the expectations for Fe nuclei.

35th International Conference of High Energy Physics - ICHEP2010,

July 22-28, 2010

Paris France

\footnotetext{
* Speaker.

$\dagger$ †ull author list available at http://www.auger.org/archive/authors_2010_11.html
} 


\section{Introduction}

The Pierre Auger Observatory is located near the town of Malargüe in Argentina. It started data taking in January 2004 with only 100 water Cherenkov detectors and one fluorescence telescope installed [1]. The baseline configuration comprising 1600 water Cherenkov detectors and 24 fluorescence telescopes overlooking the site of more than $3000 \mathrm{~km}^{2}$ was reached in mid 2008. Already by the end of 2009 a total exposure of about twenty thousand $\mathrm{km}^{2} \mathrm{sr}$ yr had been accumulated, much larger than that of all previous air shower experiments combined. The Pierre Auger Observatory is the first hybrid air shower experiment combining two independent observation techniques complementing each other. The surface detector (SD) has a duty cycle of almost $100 \%$, collecting the vast majority of data which are used for spectrum measurements and anisotropy searches. The fluorescence detector (FD), on the other hand, can be operated only during clear moonless nights reaching a duty cycle of about $15 \%$. The FD enables the longitudinal development in the atmosphere to be measured. This provides a calorimetric and thereby basically model independent measurement of the cosmic ray energy. For this subsample of events, a cross calibration between the two detection techniques can be performed and then be applied to the large data set of the surface detector array. Moreover, the fluorescence measurements enable the determination of the depth of maximum development of the shower, which encodes information about the composition of the primaries and about the properties of the first hadronic interactions. The Pierre Auger Observatory has already enabled us to begin addressing many pressing questions of the highest energy cosmic rays that motivated its construction. This includes measuring the features present in the spectrum, searching for anisotropies in the cosmic ray arrival directions distribution or constraining the composition of the primary cosmic rays.

\section{Energy Spectrum}

Measuring the ultra-high energy cosmic ray (UHECR) energy spectrum is a key part in the quest for understanding the origin of these particles, reaching energies of $10^{8}$ times higher than those accelerated in the LHC. At present, the most reliable measurement of the primary energy is given by observation of the fluorescence emission in the atmosphere. Laboratory measurements have determined the absolute yield of fluorescence photons per unit of energy deposit as well as their emission spectrum and dependence on atmospheric parameters (see e.g. [2]). Once the shower axis with respect to the telescopes is reconstructed from the projected light trace and timing of the telescope pixels and water tanks, the absolute light intensity as a function of atmospheric depth $X$ can be calculated. The total energy deposited in the atmosphere is then given by the integral of $d E / d X$ over depth. While conceptually simple, the implementation of the technique is somewhat more complex. For details the reader is referred to e.g. [3]. The SD analysis starts with a reconstruction of the shower arrival direction from the relative timing of the SD stations and proceeds with a fit of the lateral distribution of particle densities. Here, the signal $S(1000)$ obtained $1000 \mathrm{~m}$ from the shower core yields an energy estimator which is insensitive to shower fluctuations and the unknown primary mass. Obviously, $S(1000)$ will depend on the amount of atmosphere traversed by the shower. However, this attenuation can be accounted for based on experimental data so that the measured value $S(1000)$ can be related to that expected at a chosen nominal zenith angle, here $\theta=38^{\circ}$. This quantity, $S_{38^{\circ}}$, measured in units of vertical equivalent muons (VEM) 

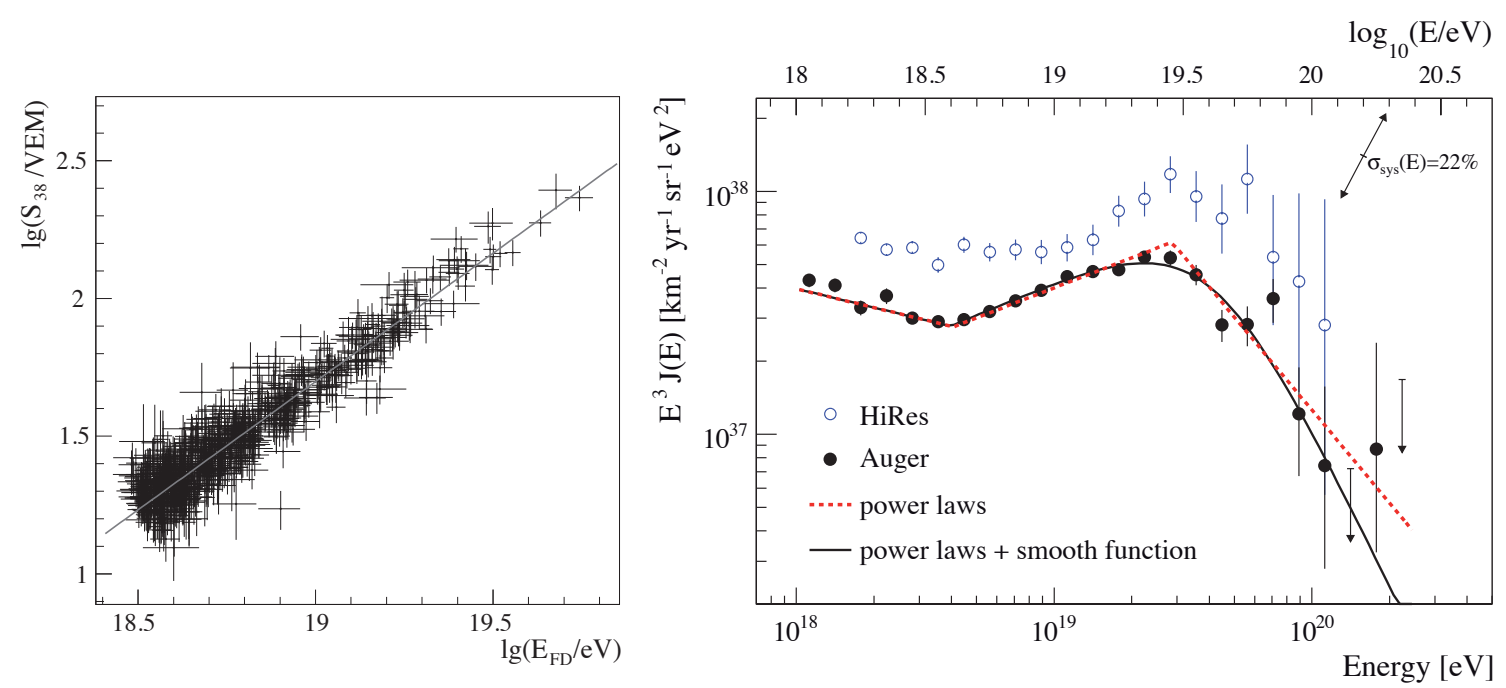

Figure 1: Left: FD energies from a sample of 795 high quality hybrid events are used to calibrate the SD energy parameter $S_{38^{\circ}}$. Right: Combined hybrid and SD energy spectra from Auger [4] compared to the stereo spectrum from HiRes [5].

seen in the SD stations, is plotted in Fig. 1 (left) with respect to the absolute energy reconstructed from the FD. A fit of this correlation function then provides the energy calibration of the SD.

The derived energy spectrum is presented in Fig. 1 (right). Simple power law fits indicate that the ankle is located at $\log (E)=18.61 \pm 0.01$ and the spectral break at $\log (E)=19.46 \pm 0.03$ with the power law indices being $\gamma=3.26 \pm 0.04,2.59 \pm 0.02$, and $4.3 \pm 0.2$ for the different regions, respectively. Within uncertainties of about $20 \%$ for the determination of the primary energy, the Auger and HiRes spectrum can be considered consistent. The traditional interpretation of a spectral suppression at this energy is the GZK-effect [6], but one should note that heavy particles also lose energy in the CMB via photo-disintegration. While it is highly suggestive to interpret the observed suppression as being due to CR energy losses in the CMB it should be noted that nearby sources reaching their limiting energy could mimic a similar spectral feature. This question is hoped to be answered by measurements of the photon and neutrino fluxes expected from the GZK effect.

\section{Arrival Directions}

Searches for anisotropies in the arrival directions are motivated by the fact that CR trajectories in galactic and extragalactic magnetic fields become straighter as the energy increases. This gives the hope that CR astronomy may become feasible at ultra-high energies. Moreover, the GZK effect limits the horizon from which UHECR can be observed to less than about $100 \mathrm{Mpc}$. This implies that only sources within a relatively close-by neighborhood can contribute to the fluxes observed at Earth. One class of potential sources that may be able to accelerate particles up to these extreme energies are Active Galactic Nuclei (AGN), consisting of supermassive black holes with masses up to $\sim 10^{9} M_{\odot}$ accreting matter in the center of galaxies and emitting powerful jets. An analysis performed by the Auger Collaboration indeed established a correlation with the AGN in the Véron Cetty and Véron (VCV) catalog [7]. This correlation was most significant for events above $55 \mathrm{EeV}$ and angular separations of less than $3.1^{\circ}$ from AGN closer than $75 \mathrm{Mpc}$. In the latest study with 


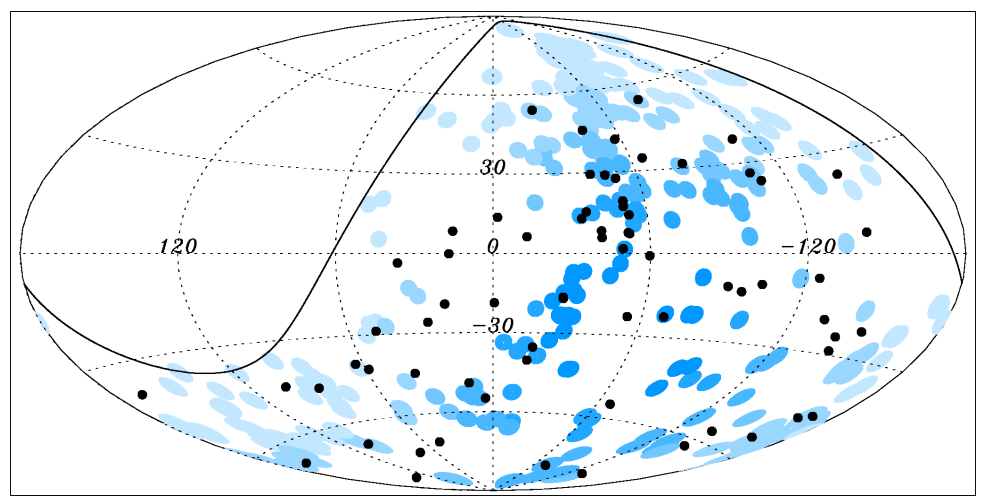

Figure 2: Arrival directions of the events above $55 \mathrm{EeV}$ (dots) and $3.1^{\circ}$ circles around the directions towards AGN in the VCV catalog closer than $75 \mathrm{Mpc}$ [8]. In this map, Cen A is located at $l \simeq-50.5^{\circ}, b \simeq 19.4^{\circ}$.

data up to the end of 2009, the fraction of events correlating within those parameters (excluding the events from the initial period used to fix those values) is $38_{-6}^{+7} \%$, well above the $21 \%$ that would be expected if the distribution were isotropic [8]. A map of the observed arrival directions (dots) in galactic coordinates is shown in Fig. 2, which also displays circles of $3.1^{\circ}$ radius around the location of nearby VCV AGN. One finds that 29 out of the 69 events do indeed fall inside one of the circles. Note that due to obscuration effects the catalogs are particularly incomplete near the galactic plane, and hence it is understandable that most of the events within $10^{\circ}$ of the galactic plane do not correlate with objects in the catalog. Alternative studies employing different catalogs and methods avoiding the use of specific correlation angles between UHECR and source objects yield similar results and are reported in [8].

A significant concentration of events is found around the location of Centaurus A, which is particularly interesting because this AGN lies at only $\sim 4 \mathrm{Mpc}$ from us. Inspecting the number of observed events as a function of the angular distance from the Cen A nucleus shows a most significant departure from isotropy for $18^{\circ}$, for which 13 events are observed while only 3 are expected from an isotropic distribution. Whether these events come from Cen A or from other sources, such as from the Centaurus cluster lying behind (at $\sim 45 \mathrm{Mpc}$ ) is still unclear, but this is certainly a region that looks especially promising for future anisotropy searches.

\section{Mass Composition}

Knowledge of the mass composition is another key component in understanding the origin of UHECR. Particularly, possible changes as a function of energy would help to better understand the origin of the spectral features observed in Fig. 1.

Information about the mass and type of a primary particle can be inferred from various observational features of extensive air showers, most importantly from the electron/muon ratio of particles at ground and from measurements of the depth of maximum development of the shower in the atmosphere. For example, a primary proton of given energy will penetrate much deeper into the atmosphere than an Fe nucleus of the same total energy and will lead to much larger fluctuations on shower-by-shower basis. This follows from very basic considerations and is quantified by detailed EAS simulations. The two related observables accessible to fluorescence telescopes are $X_{\max }$ 

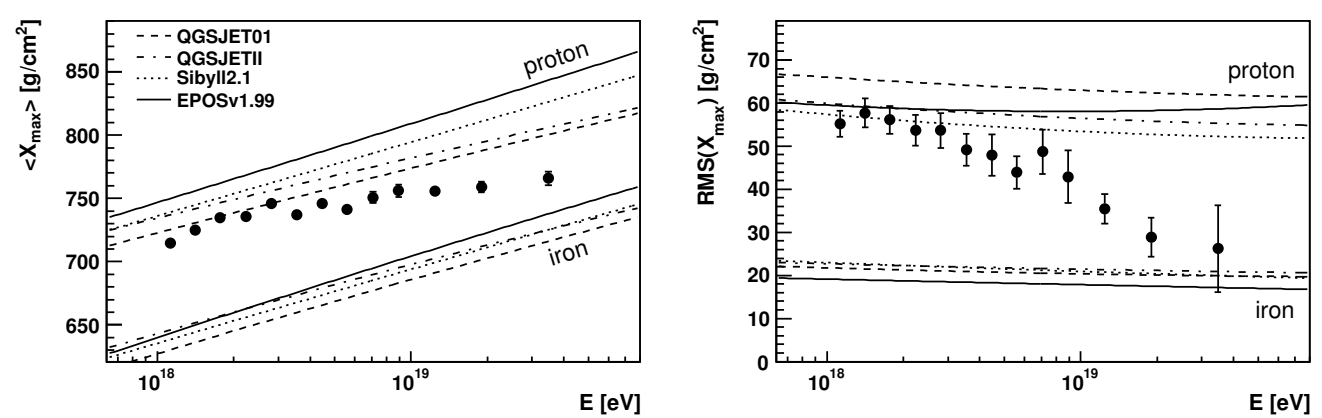

Figure 3: Measurements of $X_{\max }$ (left) and its RMS (right) as a function of energy [9].

and its fluctuations. The results of such measurements are presented in Fig. 3 together with predictions for proton and Fe primaries using different hadronic interaction models [9]. These models need to extrapolate the features of hadronic interactions well beyond the cms-energies accessible at man-made accelerators. Their uncertainties are correspondingly large and the wide distribution of predictions in the figure demonstrates that the systematic uncertainties in this analysis can be significant.

With this caveat kept in mind, a transition from a light composition up to the ankle approaching the expectations for heavier nuclei up to $40 \mathrm{EeV}$ is inferred from both the $\left\langle X_{\max }\right\rangle$ values and from its RMS values. Unfortunately, FD data statistics do not yet allow the extension of these measurements into the the GZK domain, where the onset of directional correlations is observed. An Fe dominated composition to beyond $55 \mathrm{EeV}$ would indeed be challenging to understand in combination with the reported observations of directional correlations with AGN. One needs to note here that an increase of the p-nucleus inelastic cross-section beyond what is adopted in the interaction models could mimic the expectations for heavier nuclei, but simultaneous description of $\left\langle X_{\max }\right\rangle$ and its RMS would be difficult to reach. Thus, LHC data in the forward region will be of great importance to improve the reliability of the interaction models. This, together with better statistics, particularly of composition and anisotropy measurements at higher energies will help to unravel the puzzles of cosmic rays at the highest energies.

Acknowledgement: KHK would like to thank the German Ministry for Research and Education (BMBF) for financial support.

\section{References}

[1] J. Abraham et al. [Pierre Auger Coll.], Nucl. Instr. Meth. A523 (2004) 50.

[2] M. Monasor et al., Astropart. Phys. 34 (2011) 467.

[3] M. Unger et al., Nucl. Instr. Meth. A588 (2008) 433.

[4] J. Abraham et al. [Pierre Auger Coll.], Phys. Lett. B685 (2010) 239; Phys. Rev. Lett. 101 (2008) 061101.

[5] R. Abbasi et al. [HiRes Collaboration], Astropart. Phys. 32 (2009) 53.

[6] K. Greisen, Phys. Rev. Lett. 16 (1966) 748, and G.T. Zatsepin and V.A. Kuz'min, Sov. Phys. JETP Lett. (Engl. Transl.), 4 (1966) 78.

[7] J. Abraham et al. [Pierre Auger Coll.], Science 318 (2007) 938; Astropart. Phys. 29 (2008) 188.

[8] J. Abraham et al. [Pierre Auger Coll.], Astropart. Phys. 34 (2010) 314.

[9] J. Abraham et al. [Pierre Auger Coll.], Phys. Rev. Lett. 104 (2010) 091101. 